\title{
XVII.
}

\section{Die Behandlung des Freiheitsproblems bei John Locke.}

\author{
Von
}

Dr. A. Messer, Giessen.

Fortsetzung (s. Bd. XI, S. 133-149).

3. An erster Stelle ergab sich uns also bei unserer Betrachtung der termini eine, wohl nur auf einer gewissen Ungenauigkeit beruhende, Entgegensetzung der Denkkraft $=$ Verstand und der Bewegungskraft $=$ Wille, eine Entgegensetzung, die der Scheidung des geistigen und körperlichen Gebiets und der ihnen angehörigen Thätigkeiten entsprach, und aus dieser sich wohl herleitete.

Neben dieser Unterscheidung begegnete uns eine andere, die wohl in zutreffenderer Weise Lockes wirkliche Anschauung zum Ausdruck bringt. Hierin werden gegenübergestellt: Erkenntniskraft und Kraft des Wollens, Verstand und Wille; beide dem geistigen Gebiet angehörig, beide auf das körperliche hinüberreichend: der Verstand allerdings nur „leidend“, insofern er die Fähigkeit hat, Vorstellungen durch die Wirksamkeit äusserer Substanzen zu empfangen (II, 21, §72); der Wille „thätig“, insofern er körperlịche Bewegungen zu veranlassen oder zu hemmen vermag; daneben erstreckt er seine Wirksamkeit auch auf den Verstand, indem er die Betrachtung oder Nicht-Betrachtung von Vorstellungen anordnet ${ }^{38}$ ).

38) Dabei ist aber zu beachten, dass, wenn einmal das Erkenntnisvermögen in Thätigkeit gesetzt ist, die Art des Erkennens nicht mehr von unserem Belieben abhängt, sondern durch die Gegenstände bestimmt wird, soweit sie klar erfasst werden. Locke führt dies im 13. Kap. des IV. Buches näher aus. 
Noch in einer anderen Beziehung zeigt sich bei Locke ein Schwanken in der Abgrenzung dessen, was als Wirkungsbereich des Willens bezeichnet wird, und damit in der Fassung dieses Begriffes überhaupt.

Er gebraucht vielfach die Ausdrücke power of preferring (z. B. II, 21, §6) oder power to prefer or choose (z. B. II, 21, § 17; $\S 18 ; \S 19)$ in der Bedeutung von will und demgemäss auch preference in der Bedeutung von volition (z. B. II, 21, §9; §27).

Nun kommt er aber auch zur Erörterung der Frage, wodurch der Wille bestimmt werde, oder, wie er sie genauer definirt: was die Seele veranlasse in jedem einzelnen Fall ihre allgemeine leitende Kraft (its general power of directing II, 21, § 29 d. i. eben "den Willen") zu dieser besonderen Bewegung oder Ruhe zu bestimmen. Hierbei erklärt er (II, 21, § 30), dass Ausdrücke wie „Wählen", „Vorziehen" (choosing, preferring) ungenau ue Bezeichnungen des "Willens" (willing, volition) seien, weil sie zugleich auch für "Wünschen“ (desire) passten. „Wünschen“ müsse aber bestimmt von "Wollen". unterschieden werden, was schon daraus hervorgehe, dass Wille und Wunsch bisweilen nach entgegengesetzter Richtung gingen ${ }^{39}$ ). "Wille" sei also nur da vorhanden, wo die Seele durch blosses Denken eine Handlung anzufangen, fortzusetzen oder damit aufzuhören unternehme, von der sie voraussetzt, dass sie in ihrer Macht stehe (the will or power of volition is conversant about nothing, but that particular determination of the mind, whereby barely by a thought the mind endeavours to give rise, continuation, or stop, to any action which it takes to be in its power II, 21, $\S 30$ ) oder, wie es an einer anderen Stelle ${ }^{40}$ ) heisst: „das Wollen ist offenbar ein Thun der Seele,

39) Er erläutert dies durch folgendes Beispiel: „A man whom I cannot deny, may oblige me to use persuasions to another, which, at the same time I am speaking, I may wish may not prevail on him. In this case, it is plain the will and desire run counter. I will the action that tends one way, whilst my desire tends another, and that the direct contrary way. (II, $21 \S 30$.)

40) II, 21, § 15. Ich merke auch das Vorausgehende hier an: „I must here warn my reader that ordering, directing, choosing, preferring etc. which I have made use of, will not distinctly enough express volition, unless he will reflect on what he bimself does when he wills. For example, preferring, 
die wissentlich die Herrschaft ausübt, die sie über jeden Teil des Monschen in Anspruch nimmt".

So unterscheidet also Locke, wo er genau die von ihm gewollte Auffassung dieses Begrifies darlegt, in dem "Wollen" zwei Momente: 1. ein "Vorziehen", 2. die Ausübung der Macht, die die Seele über den Menschen besitzt (oder wenigstens die mit der Intention dieser Ausübung verbundene Voraussetzung, dass dieselbe möglich sei).

Diese Fassung des Begriffes "Wille" verschiebt sich aber an den Stellen, wo Locke den Begriff „Freiheit“ mit in Erörterung zieht und es unternimmt, diesen letzteren von jenem reinlich zu scheiden.

Die Vorstellung der Freiheit (und Notwendigkeit), so führt er aus, entsteht durch Selbstwahrnehmung, die sich auf die Bethätigung unseres Willens richtet. „Da alle Thätigkeit sich auf Denken und Bewegen beschränkt, so ist ein Mensch insofèrn frei, als er die Kraft hat, je nachdem seine Seele es vorzieht oder bestimmt, zu denken oder nicht zu denken, zu bewegen oder nicht zu bewegen", (II, 21, § 8): eben darin besteht aber, wie wir oben gesehen haben, die Willensbethätigung.

Er setzt darauf näher aus einander, dass Freiheit A) Verstand und Willen voraussetzte, dass sie aber B) damit noch nicht gegeben sei, da durch 1) äussere oder 2) innere Gründe die Selbst-

which seems perhaps best to express the act of volition, does it not precisely. For though a man would prefer flying to walking, yet who can say he ever wills it? Volition, it is plain, is an act of the mind knowingly exerting that dominion it takes itself to have over any part of the man, by employing it in, or withholding it from, any particular action. Da Handlung (action) entweder in einem Denken oder in einem Bewegen besteht, so ergeben sich zwei Arten von Willenshandlungen: Bewegungen der körperlichen Organe oder Veränderungen der geistigen Processe. Dabei wird übrigens - ganz anders als wie bei Spinoza, aber entsprechend auch den erkenntriistheoretischen Ansichten Lockes - ein Hinübergreifen des rein psychischen Willensaktes in das physische Gebiet vorausgesetzt. Doch hebt Locke gelegentlich hervor, die Mitteilung der Bewegung von einem Körper auf den andern sei „ebenso dunkel und unbegreiflich, wie ảe Art, auf welche die Seele ihren Körper bewegt oder zur Ruhe bestimnt; obgleich es jeden Augenblick geschieht“. (II, 23, § 28, cf. IV $, 7, \S$ 10.) 
macht a) über unseren Körper und b) über unser Denken aufgehoben werden könne ${ }^{41}$ ).

Frei ist also ein Wesen, insofern es 1) denkend und wollend oder, wie wir in Anlehnung an die kürzere Ausdrucksweise Lockes (z. B. in der zuletzt angeführten Stelle II, 21, § 8) sagen können, insofern es Träger "thätiger Kraft" d. i. „wollend" ist, und insofern es 2) nicht durch innere oder äussere Gründe an der Bethätigung dieser Kraft gehindert ist. Das erste Moment würde sich als die poșitive, das zweite als die negative Seite der Freiheit bezeichnen lassen.

Nach ihrer positiven Seite würden die Bezeichnungen "frei" und "(denkend und) wollend" zusammenfallen, so dass in der That, die Frage, ob der Wille frei sei, identisch wäre mit der Frage, ob die Freiheit frei sei - wie Locke dies ausdrücklich gelegentlich hervorhebt ${ }^{42}$ ).

Nach ihrer negativen Seite aber könnte die „Freiheit" ganz wohl vom "Willen" unterschieden werden.

Nun sucht aber Locke die „Freiheit" auch nach ihrer positiven Seite hin (also nicht bloss in der Bedeutung des Freiseins von

${ }^{41}$ ) Alle diese Fälle erläutert er durch Beispiele (II, 21, § 9 und § 12). Ad A. Ein fliegender Ball ist nicht frei, weil er „kein Denken und folglich kein Wollen" hat; ad B. 1a. Ein von einer brechenden Brücke ins Wasser fallender Mensch ist hierbei nicht frei (§9); ad B. 1b. Jemand, der infolge einer krampfhaften Bewegung seines Armes seinen Freund schlägt, ist hierin nickt frei (\$9); ad B. 2 a. „Ein Mann auf der Folter ist nicht frei in Beseitigung der Vorstellung des Schmerzes und in Beschäftigung der Seele mit andern Gedanken" ( $\$ 12)$; ad B. 2b. Eine aufbrausende Leidenschaft nimmt uns die Freiheit anderes zu denken (§12). - In einem Excurs (§10 u. $\S 11)$ wird darauf hingewiesen, dass die Freiheit auch dann aufgehoben ist, wenn die Richtung, nach der äussere oder innere Gründe unser Verhalten zwingend bestimmen, zufällig mit der Richtung unseres Willens zusammenfällt.

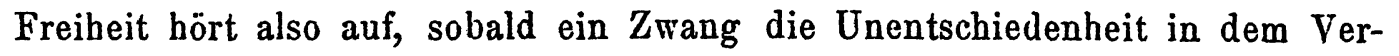
mögen $\mathrm{zu}$ handeln oder nicht $\mathrm{zu}$ handeln (that indifferency of ability on either side to act or to forbear acting) aufhebt (\$10).

42) II, 21, § 16. If freedom can with any propriety of speech be applied to power, or may be attributed to the power that is in a man to produce or forbear producing motion in parts of his body, by choice or preference; which is that which denominates him free, and is freedom itself. But if any should ask whether freedom were free, be would be suspected not to understand well what be said.

Archiv r. Geschichte d. Pbilosophie. XI. 3. 
Hemmnissen) von dem; Willen" $z u$ unterscheiden und sie als ein (positives) "Vermögen" dem Menschen beizulegen ${ }^{43}$ ). Er sagt geradezu: „Es ist also klar, dass der Wille nur eine Macht oder ein Vermögen und die Freiheit eine andere Macht oder Vermögen ist" (II, 21, $\$ 16:$ It is plain then, that the will is nothing but one power or ability; and freedom another power or ability).

Aber während Locke zu dieser Unterscheidung von Wille und Freilheit hinstrebt, verändert sich ihm, ohne dass er es merkt, unter der Hand der Begriff des Willens, wie dies ganz deutlich der Gedankengang des vorausgehenden $\$ 15$ zeigt.

Am Anfange dieses $\S 15$ hatte er die oben dargelegte Definition des Wollens in der Art gegeben, dass sich darin zwei Momente unterscheiden liessen: .1) das Vorziehen, 2) die Ausübung. der Selbstmacht diesem Vorziehen entsprechend. - Locke fährt darauf fort" ${ }^{44}$ ): „Kann man leugnen, dass jedes Wesen, das die Kraft hat, an sein eignes Hàndeln zu denken und dessen Ausführung oder Unterlassung vorzuziehen, das Vermögen besitzt, was man Willen nennt? Der Wille ist deshalb nur eine solche Kraft. Freiheit ist dagegen die Kraft eine einzelne ${ }^{45}$ ) Handlung zu thun

43) Wir können hier noch gänzlich absehen von dem, was sich für Locke im Laufe seiner Untersuchung als Inhalt dieses Vermögens der Freibeit (oder richtiger gesagt: der wahren Freiheit) herausstellt, nämlich der Fähigkeit, den Resultaten vernünftiger Ueberlegung entsprechend zu handeln.

44) II, 21, § 15: „For can it be denied, that whatever agent has a power to think on its own actions, and to prefer their doing or omission either to other, has that faculty called will? Will then is nothing but such a power. Liberty, on the other side, is the power a man has to do or forbear doing any particular action, according as its doing or forbearance has the actual preference in the mind; which is the same thing as to say, according as he himself wills it.

45) Wenn hier die „Freiheit" als die Kraft bezeichnet wird, eine einzelne Handlung zu thun oder zu unterlassen, so könnte sich die Vermutung aufdrängen, Locke wolle etwa mit „Wille“ jene Kraft im allgemeinen, abgesehen von ihren einzelnen Bethätigungen, bezeichnen. Diese Vermutung erweist sich aber als unzutreffend; denn gerade umgekehrt erklärt er II, 21, $\S 71$ „Wille" als „die Kraft, welche die wirkenden Vermögen in dem e inz elnen Falle in Bewegung oder Ruhe versetzt". (A power to direct the operative faculties to motion or rest in particular instances, is that which we call the will. Unmittelbar vorher steht: „Liberty is a power to act or not to act, according as the mind directs"). 
oder zu unterlassen, je nachdem der Mensch das Eine oder das Andere vorzieht, was ebenso viel heisst, als je nachdem er es will" - worauf dann die erwähnte Scheidung zwischen den beiden „Vermögen": Willen und Freiheit folgt:

Ganz augenscheinlich ist jetzt das zweite Moment aus dem Willen eliminiert; eben es (d. i. jene "Selbstmacht") ist jetzt als "Freiheit" bezeichnet; diese ist also zu einem positiven Vermögen geworden, während der Wille auf seinen ersten Bestandteil eingeschränkt ist; was Locke unwillkürlich dadurch anerkennt, dass er die Identification von "Wollen" und "Vorziehen", die er am Anfange eben dieses $\S 15$ als ungenau bezeichnet hatte; sofort selbst wieder einsetzt.

Dass ihm aber diese Begriffsverschiebung entgeht, wird dadurch erleichtert, dass er, wie schon oben bemerkt wurde, durchgängig "Wollen" und „Vorziehen“ (oder „Wählen“) als gleichbedeutend anwendet.

Während er das Ungenaue dieses Sprachgebrauchs nur an zwei Stellen (II, 21, $\S 15$ und $\S 30$ ). hervorhebt, gebraucht er dagegen den Begriff „Freiheit“ (liberty) meist in der dargelegten Bedeutung einer (positiven) Eigenschaft (z. B. II, 21, § 8; §21; 71). -

Lässt sich nun nicht doch, auch ohne Vermischung mit dem Willen, dem Begriffe Freiheit ein positiver Inhalt geben, der Lockes Sprachgebrauch wenigstens in einem bestimmten Sinne rechtfertigte? - Während Locke vielfach die wirkliche Ausführung der willkürlichen Handlungen (psychischen und physischen) dem Willen selbst zuschreibt, redet er an anderen Stellen. (z. B. II, 21, § 71) davon, dass der Wille die wirkenden Kräfte des Menschen (the operative faculties or powers of the man) in Bewegung oder Ruhe versetze, so dass also diese die Handlung selbst ausführen, und der Wille nur den Impuls dazu gebe. Mit diesen operative powers kann er nur meinen: Die Bewegungskraft (motivity, die er II, 23, $\S 18$ ungenau mit dem Willen selbst gleichsetzt) und die Denkkraft (perceptivity or power of perception or thinking II, $27, \S 73$ ). Indem nun diese Vermögen den nach dieser oder jener Richtung wirkenden Willensimpulsen zugänglich sind, zeigen sie eine Art „Unentschiedenheit" (indifferency). „So habe ich z. B. 
das Vermögen, meine Hand zu bewegen oder sie ruhen zu lassen; diesor wirkonden Kraft (operative power) ist es gleichgültig, ob sie moine Hand bewegt oder nicht, und ich bin deshalb in dieser Bezichung vollkommen frei. Bestimmt mein Wille diese Kraft zur Ruhe, so bleibe ich doch frei, weil diese Gleichgültigkeit jener Kraft für das Handeln oder Nichthandeln immer bleibt" (II, 21, § 71). Locke erklärt nun ausdrücklich, dass er mit Freiheit (liberty) eben diese Indifferenz der wirkenden Vermögen (indifferency of the operative powers of the man) meine. Also hätte der Freiheitsbegriff doch einen, von dem, Willen unterscheidbaren, positiven Inhalt: er bedeutet nicht nur die Abwesenheit von Momenten, die jene Indifferenz und damit die Macht des Willens über den Vorstellungs- und Bewegungsmechanismus aufheben, sondern auch jene Indifferenz selbst. Es ist jedoch zu beachten, dass diese Erklärung des Begriffs der Freiheit durch den der Indifferenz erst in den späteren Auflagen eingefügt ist.

Die oben aufgewiesene Unklarheit hat augenscheinlich folgenden Grund. Locke ist bestrebt die Begriffe Wille und Freiheit als gar nicht zusammengehörig zu scheiden. Einer seiner Beweisgründe ist auch der: beides seien Kräfte (powers), diese könnten aber nicht einander zukommen, sondern nur einer Substanz. Solche Stellen erwecken den Eindruck, als liesse sich das Wort power ganz in demselben Sinne für die Freiheit anwenden wie für den Willen, als sei auch sie nicht sowohl die Art und Weise einer wirkenden Kraft (etwa die "Indifferenz"), sondern selbst ein aktives. Vermögen. -

IV. Die Behandlung des Freiheitsproblems im Essay concerning human understanding.

1. Die Erörterung über die Freiheit ist eingeschoben in das 21. Kapitel des 2. Buches, das von der Vorstellung ; Kraft" (power) handelt, und füllt hier die $\$ \S 7-73$.

Die Vorstellung "Kraft" gehört zu jenen einfachen Vorstellungen (simple ideas), welche durch Sensation und Reflexion erlangt werden, denn die Seele erhält sie durch Wahrnehmung in sich und ausser sich $(\$ 1)$. 
Als Veränderung bewirkend heisst sie "thätige", als solche erleidend „leidende Kraft" (active und passive power) (\$2).

Sie ist eine einfache Vorstellung, obwohl sie eine Beziehung einschliesst und zwar auf Thätigkeit ( $\$ 3$ ).

Die Bezeichnung der Vorstellung "Kraft" als einer "einfachen "Vorstellung erscheint auffällig. Locke hat nämlich schon in c. 2-11 des II. Buches die einfachen Vorstellungen behandelt, bei deren Aufnahme die Seele sich nur "leidend " verhält (II, 12, $\S 1$ ), er schliesst daran in c. $12-28$ die Erörterung der zusammengesetzten Vorstellungen (complex ideas) an, welche die Seele aus den einfachen selbst bildet (l. c.). Die Vorstellung der Kraft und die damit zusammenhängende der Freiheit, deren Besprechung das c. 21 füllt, wird also unter den complex ideas behandelt (und zwar unter den simple modes; denn die complex ideas zerfallen ja bekanntlich in die Vorstellungen der Modi (c. 12-22), der Substanzen (c. 23. 24) und Relationen (c. 25-28); die Modi wieder in einfache (c. 13-21) und gemischte c. 22)./So heisst es z. B. auch bei R. Falckenberg, Geschichte der neueren Philosophie (2. Aufl. 1892) S. 130: „Durch willkürliche Kombination der einfachen Vorstellungen (simple ideas) entstehen die zusammengesetzten (complex ideas)“; und unter den simple modes wird (S. 131) auch die Vorstellung power (Vermögen, Kraft) erwähnt. Diese wäre also eine complex idea. Nun wird sie aber hier (und auch z. B. II, 22, § 10) ausdrücklich als simple idea bezeichnet. Das ist doch augenscheinlich ein Widerspruch. Aber noch ein anderes Bedenken erhebt sich hier. Locke hat vorher (II, $12, \S 5$ ) von den simple modes die Definition gegeben: „Sie sind nur Abwechselungen oder Verbindungen einer und derselben einfachen Vorstellung, ohne dass andere ihr zugemischt werden z. B. ein Dutzend, ein Schock; es sind dabei eine gewisse Menge Einheiten nur zusammengerechnet". Wie sollte sich aber diese Definition auf die Vorstellung "Kraft" anwenden lassen? -

$W_{0}$ die Lösung diesser Schwierigkeiten zu suchen ist, ergiebt sich aus der Stelle, an der Locke die Besprechung der simple modes beginnt (II, 13, $\S 1$ ). Er führt dort aus, er habe die einfachen Vorstellungen bis jetzt mehr nach dem Wege be- 
trachtet, auf dem sie in die Seele gelangten, als nach ihrem Untcrschied von den mohr zusammengesetzten Vorstellungen. Es sei deshalb zweckmässig, "einige davon noch einmal unter dem letzten Gesichtspunkt zu betrachten und die verschiedenen Besonderungen derselben Vorstellung zu prüfen, welche die Seele entweder in den bestehenden Dingen antrifft" oder selbst errougen kann. Hier wird also die oben angeführte Erklärung, dass die Seele die simple modes (wie überhaupt alle complex ideas) selbst herstelle, zum Teil zurïckgenommen: die Seele nimmt sie auch teilweise lediglich passiv auf wie die simple ideas.

Wenn man ferner zusieht, welche Vorstellungen Locke in den folgenden Kapiteln unter den simple modes behandelt, so ergiebt sich ganz klar, dass er hiermit nicht nur von der Seele selbst erzeugte Vorstellungen (wie etwa die mathematischen Grössen) meinen kann, sondern dass er darunter auch solche Modificationen (d. h. concrete Ausgestaltungen) einfacher Vorstellungen versteht, welche die Seele als gegebene lediglich passiv aufnimmt (natürlich im Sinne Lockes, denn für die auch hier wirkende psychische Aktivität hat er ja noch keinen Blick): Das gilt z. B. von den Modifikationen des Tons, der Farbe und des Geschmacks (II, 18), von den Modifikationen des Denkens (II, 19. Vergl. § 1: „die Seele bemerkt eine mannigfache Besonderung des Denkens und erhält dadurch unterschiedene Vorstellungen) und denen der Lust und des Schmerzes (II, 20); das gilt aber auch in gleicher Weise von der Vorstellung der Kraft und den daraus entspringenden (II, 21, §7) der Freiheit und Notwendigkeit.

Es liegt also hier offenkundig eine Ungenaugkeit Lockes vor. Wenn er erklärt hatte, dass die Seele bei der Bildung aller modes (als complex ideas) sich aktiv verhalte, so sehen wir jetzt, dass dies für die simple modes zum grossen Teil nicht zutrifft, und dass diese also insoweit mit den simple ideas zusammenfallen. Locke selbst hat sie denn auch gelegentlich (z. B. II, $21, \S 3 ; 22, \S 10)$ ausdrücklich so bezeichnet, und auch an der Stelle, wo er zu den mixed modes übergeht, spricht er in einer Weise, als habe er sich seither lediglich mit passiv aufgenommenen Vorstellungen beschäftigt (II, 22, § 2). 
Die Sache liegt also so: Locke behandelt die Vorstellung "Kraft" unter den simple modes (also unter den complex ideas), thatsächlich aber betrachtet er sie als eine simple idea, die die Seele ganz passiv empfängt. -

Dass aber auch dies nicht ganz zutrifft, drängt sich ihm selbst auf. Er bemerkt nämlich (II, 21, §3), die Kraft könne als einfache Vorstellung gelten, obwohl sie eine Beziehung (nämlich auf Thätigkeit und Veränderung) einschliesse. Dem Scharfblick Humes ist dies nicht entgangen. An der Stelle seines Enquiry concerning himan understanding, wo er darzulegen sucht, dass weder der äussere noch der innere Sinn die Vorstellung "Kraft" liefere, bemerkt er (Essays, London 1784, vol. II. p. 68. Kirchmanns Uebersetzung S. 59A.): Mr. Locke in his chapter of power, says, that, finding from experience, that there are several new productions in matter, and concluding that there must somewhere be a power capable of producing them, we arrive at last by this reasoning at idea of power [cf. Locke, II, 21, §1]. But no reasoning can ever give us a new, original, simple idea; as this philosopher himself confesses. This, therefore, can never be the origin of that idea. -

Wir fahren nach dieser Abschweifung in der Darlegung des Gedankengangs von c. 21 fort. Die Vorstellung "Kraft" bezieht sich also auf Thätigkeit. Thätigkeiten giebt es zwei: Denken und Bewegen. Vom Denken geben uns die Körper gar keine Vorstellung, ebensowenig von einer spontanen Bewegung: also gelangt die Seele, genau genommen, zur Vorstellung der "thätigen Kraft" nur durch Wahrnehmung ihrer eignen Thätigkeit, durch Reflexion (§4).

Dabei findet sie zwei Kräfte in sich wirksam: den Willen (will) und die Auffassungskraft oder den Verstand (power of perception oder understanding) ( $(5)$.

Die Erwähnung des Verstandes unterbricht hier etwas den Gedankenfortschritt. Denn am Schlusse des $\S 4$ war bemerkt worden, die Seele erlange deshalb durch Selbstwahrnehmung die Vorstellung der "thätigen Kraft", weil sie in sich eine Kraft wahrnehme, die ein Thun: Bewegen oder Denken anfange $\left.{ }^{46}\right)$. Es fragt

46) The power to begin any action, either motion or thought. 
sich nun: welches ist dieso Kralt? Dio Antwort darauf giebt $\S 5$ im orsten Teil: diese Kraft der Seele ist der Wille.

Der Gedankongang führt nun erst im $\$ 7$ veiter: aus der Beobachtung des Wirkungsbercichs dieser Seelenkraft entstelien die Vorstellungen "Freihcit" und "Notwendigkeit" (From the consideration of the extent of this power of the mind over the actions of the man, which every one finds in himself, arise the ideas of liberty and necessity). Es kann also die Bemerkung über den Verstand $(\$ 5,2$. Teil) und die - später wiederholte - Mahnung, durch den Ausdruck "Seelenvermögen" (faculty of the mind) sich nicht irre leiten zu lassen ( $(6)$, für den Gedankenfortschritt ausser Betracht bleiben.

2. Die beiden Vorstellungen "Freiheit" und "Notwendigkeit" werden nunmehr an sich einer Betrachtung unterzogen, und zwar die erste in $\$ \S 8-12$, die zweite in $\S 13$.

Die Freiheit wird erklärt als "die Kraft eines Wesens, eine einzelne Handlung dem Entschlusse oder Denken der Seele gemäss $z u$ thun oder zu unterlassen, wobei eines von beiden vorgezogen wird. (A power in any agent to do or forbear any particular action, according to the determination or thought of the mind, whereby either of them is preferred to the other $\S 8$.)

Es wird dann, wie schon oben erwähnt, dargelegt, dass die Freiheit 1) Denken und Wollen voraussetzt und 2) ebenso die Abwesenheit äusserer oder innerer Gründe, die die Selbstmacht (d. i. eben die „Freiheit“, die hier - wie schon die soeben gegebene Definition zeigt - als positive Eigenschaft gefasst ist) entweder über unsere Körperbewegungen (\$9) oder über unser Denken ( $\$ 12)$ aufheben.

In einem Excurs wird darauf hingewiesen, dass auch solches, was notwendig stattfindet, gewollt (voluntary) sein kann; mithin bildet zu voluntary nicht necessary den Gegensatz, sondern involuntary. Es stehen sich also gegenüber: a) freiwillig, (free) - notwendig (necessary); b) gewollt (voluntary) - nicht gewollt (involuntary).

Locke übernimmt diese Unterscheidung wohl aus der scholastischen Philosophie, in deren Sinne V. Cathrein (Moralphilo sophie $I^{2}$ (1893) S. 41) ausführt: "Das vom Willen als Wirkung 
Gew ollte (voluntarium) ist nicht gleichbedeutend mit dem Freiwilligen (liberum). Freiwillig ist nur, was aus der freien Wahl des Willens hervorgeht und auch hätte vermieden werden können. Das Gewollte dagegen umfasst zwar auch das Freiwillige, dehnt sich aber auch auf alles aus, was mit Nothwendigkeit aus dem Willen hervorgeht." $\mathrm{Er}$ bemerkt dazu mit Recht, es fehle im Deutschen ein gleichwertiger Ausdruck für voluntarium, denn "gewollt" bedeute a) was Gegenstand unseres Wollens ist (volitum), b) was irgendwie unserem Willen das Dasein verdankt (voluntarium). - Locke hat sonst die feste und scharfe Terminologie der Schulphilosophie meist vernachlässigt - nicht zum Vorteil seiner Erörterung.

3. Bis hierher ist das eigentliche Problem der „Willensfreiheit" noch nicht berührt, mit „Freiheit" ist nur gemeint die Freiheit zu handeln, nicht die zu wollen.

Jetzt tritt er an die Frage heran: „ob der menschliche Wille frei ist oder nicht". (Whether man's will be free, or no?)

Er betont hier zunächst, diese Frage sei ebensowenig zu bejahen, als zu verneinen, sondern sie sei als unrichtig gestellt abzuweisen ( $\$ 14)$. Denn der Wille sei eine Kraft, und die Freiheit sei eine $\mathrm{Kraft}^{47}$ ) (power) ( $\$ 15$ ), die Kräfte könnten aber nicht einander angehören, sondern, als Eigenschaften (attributes), könnten sie nur selbständigen Dingen (substances) zugeschrieben werden ( $\$ 16)$.

Diese irrige Fragestellung sei entstanden durch die Einführung des Wortes „Vermögen“ (faculty), das den Willen (und den Verstand) als ein selbständiges Wesen habe erscheinen lassen ( $\$ 17-20$ ).

Die Freiheit komme also dem Wesen d.h. dem Menschen zu. „So weit jemand vermag, durch die Richtung oder Wahl seiner Seele und indem er das Dasein einer Handlung ihrem Nichtdasein vorzieht oder umgekehrt, das Dasein oder Nichtdasein derselben zu bewirken, so weit ist er frei." Denn ein solches Vorziehen einer Handlung vor ihrem Nichtsein, ist das Wollen derselben, und man kann sich kein Wesen freier vorstellen, als wenn es thun kann, was es will" ( $(21)$.

4. Mit dieser summarischen Erklärung, der Abweisung der

47) Auf diese Stelle wurde oben (S. 410) schon näher eingegangen. 
Frage nach der Willensfreiheit als oiner unrichtig gestellten, begnügt sich indessen Locke nicht. Der forschende Menschengeist, so fiihrt er aus $(\$ 22)$, beruhige sich damit nicht. Er sei bestrebt, den Gedanken der Schuld (und damit also die Verantwortlichkeit) möglichst von sich zu entfernen. Man erkläre also diese Freiheit (nämlich zu handeln, wie man wolle) sei noch nicht diejenige, die wirklich Verantwortlichkeit begründe: dazu sei vielmehr nötig, dass der Mensch ebenso frei in Wollen sei, als man ihm eine Freilicit im Handeln zuschreibe ${ }^{48}$ ).

Locke untersucht deshalb in dem ganzen folgenden Teil seiner Erörterung eingehend, in welchem Sinne man etwa doch von Willensfreiheit reden könne.

Als die zunächst sich biatende mögliche Bedeutung dieses Begriffs erscheint die Freiheit zu wollen oder nicht zu wollen, d. h. einen Willensakt zu setzen oder nicht (\$\$23, 24). Hierzu bemerkt er: „Da das Wollen ein Handeln ist und Freiheit in der Kraft zu handeln oder nicht zu handeln besteht, so ist der Mensch bezüglich der Handlung des Wollens, wenn sich eine ihm mögliche Handlung als eine gleich zu vollziehende darstellt, nicht frei“. Er muss ihr Geschehen oder Nicht-Geschehen wollen, in beiden Fällen muss er also die Handlung des Wollens vornehmen.

Ein gehender Mensch, dem vorgeschlagen wird, das Gehen zu beenden, muss sich $\mathrm{zu}$ dem einen oder andern entschliessen, er ist also darin, ob er einen Willensakt vornimmt oder nicht, nicht frei.

So verhält es sich aber mit der grössten Zahl unserer Handlungen; denn die meisten unserer täglichen Vorrichtungen treten erst an uns heran, weun sie sogleich $\mathrm{zu}$ vollziehen sind ${ }^{49}$ ). Irgend

48) Ich habe durch die breitere Umschreibung angedeutet, wie diese Stelle m. E. aufzufassen sei. Es kommt übrigens auf ihren Sinn hier, wo sie nur zur Ueberleitung zu einem weiteren Stadium der Untersuchung dient weniger an: von Wichtigkeit dagegen ist sie bei der Frage nach der Tendenz der ganzen Erörterung Lockes; es wird darum unten darauf züückzukommen sein.

${ }^{49}$ ) For considering the vast number of voluntary actions... there are but few of them that are thought on or proposed to the will, till the time they are to be done ( $\$ 24)$. Kirchmann übersetzt: „nur wenige werden bedacht oder dem Willen vorgestellt, ehe sie vollzogen. werden" richtiger wäre: ehe sie zu vollziehen sind; denn Locke nimmt an, wie das Folgende zeigt, dass sie bedacht werden, wenn auch nur kurze Zeit. 
ein Entschluss ist dabei nicht zu umgehen, mag die Betrachtung auch noch so kurz sein, und das Denken noch so schnell geschehen. Dass ein Entschluss stattfinde ist also notw endig, die Handlung freilich, zu der man sich entschliesst, ist eine voluntary ${ }^{50}$ ).

5. Darin also, dass ein Willensakt stattfinde, ist keine Freiheit, sondern die äusseren Bedingungen nötigen uns einen Entschluss in positivem oder negativem Sinne ab. Es fragt sich nun: besteht Freiheit darin, wie der Willensakt ausfällt. Ehe aber Locke hierzu gelangt, ist erst wieder eine unrichtige Fragestellung zu beseitigen.

Man pflegt nämlich die Freiheit des Handelns mit den Worten zu bezeichnen: man kann handeln, wie man will; was heissen soll: das Handeln ist nicht von gewissen ausser uns liegenden Bedingungen abhängig, sondern lediglich von unserem Willen. Da liegt es denn nahe, die sich anschliessende Frage nach der Freiheit des Wollens auch zu fassen: kann man denn wollen, wie man will? Dies ist die zweite Fassung der Frage nach der Willensfreiheit, die Locke in $\$ 25$ als eine absurde zurückweist.

Man kann diese Fassung in zwei Bedeutungen nehmen, die Locke beide streift, aber nicht klar auseinanderhält. Die erste Bedeutung entsteht so, dass man in dem ersten Satzteil: „kann man wollen" das "wollen" dasselbe bezeichnen lässt wie im zweiten Satzteil das „will“. Der Sinn wäre dann: ist es möglich so zu wollen, wie man thatsächlich will? oder umgekehrt: ist unser wirkliches Wollen möglich? - was natürlich sinnlos ist. In

50) Dies wird doch wohl der Sinn der Stelle sein; Locke lässt dabei freilich seine frühere Bemerkung (§ 11), dass der Gegensatz von voluntary nicht necessary, sondern involuntary sei ausser acht; denn hier liegt es wenigstens nahe, voluntary $=$ freiwillig $z u$ fassen. Man sehe übrigens den Text selbst: a man must necessarily will the one or the other of them, upon which preference or volition, the action or its forbearance certainly follows, and is truly voluntary (\$23). Kirchmann gelangt auffallender Weise zu der Uebersetzung: „Je nach dem Vorziehen oder Wollen folgt sicherlich die Handlung oder ihre Unterlassung, also nicht(!) wabrhaft freiwillig". Er bemerkt freilich selbst zu dieser ganzen Erörterung in $\S \S 23$ und 24: „Es ist dies eine böchst sonderbare und für den heutigen Leser $\mathrm{kaum}$ verständliche Ausführung“. (Erl. 177.) Das ist nicht der Fall; vgl. den zweiten Teil der nächsten Anmerkung. 
der zwoiten Bedoutung würde man gowissermassen zwei Sphären des Wollens annchmen. Wio man nämlich in der. Frage nach der Handlungsfreiheit (Kanu man haudoln, wie man will?) das IJandeln zurïckgeführt hat auf ein vorausliegendes Wollen, so würdo jotzt die Frage lauten: kann man so wollen, wie man will, d. h. wic ein vorausliegender Wille es bestimmt. Das würde zur Annahme eines Wollens des Wollens nötigen, womit natürlich nichts gowonnen wäre, denn sofort würde sich die Frage erheben: "Steht Dein eigenes Wollen in Deinem Willen? Und willst Du abermals dieses Wollen Deines Willens?" - wie Herbart unter Hin:weis auf diese Stelle Lockes ausführt. (Lehrbuch z. Einleitung in d. Philosophie ${ }^{2}$. § 107. A. [Sämtl. W. ed. Kehrbach. IV. 168.])

Dieser absurden Fragestellung gegenüber definiert Locke weiterhin nochmals die beiden Begriffe, die man so beharrlich auf einander beziehen will: Freiheit und Wollen ( $\$ \$ 26$ und 27).

Freiheit ist die Abhängigkeit des Seins oder Nichtseins einer Handlung von unserem darauf gerichteten Wollen und nicht die Abhängigkeit einer Handlung oder ihres Gegenteils von unserm Vorziehen ${ }^{51}$ ). Es kommt also bei der Freiheit lediglich darauf an, dass eine Handlung von uns d. h. unserm Willen abhänge. „Der auf der Klippe stehende Mensch hat die Freiheit, vierrig Fuss tief in das Meer zu springen, nicht weil er die Macht hat, das Entgegengesetzte $z u$ thun, d. h. vierzig Fuss in die Höhe zu springen, was

51) Freedom consists in the dependence of the existence, or not existence of any action, upon our volition of it; and not in the dependence of any action, or its contrary, on our preference (§27). Auch hier übersetzt Kirchmann unzutreffend: "Freiheit besteht in der Abhängigkeit des Seins oder Nicht-Seins einer Handlung von ihrem Wollen und nicht in der Abhängigkeit einer Handlung oder ihres Gegentheils von unserm Vorziehen ". Wollen und Vorziehen bilden hier keine Gegensätze, wie er durch den Druck anzudeuten scheint, sie sind vielmehr, wie so oft, ganz identisch gebraucht. - Die Scholastik unterschied nach der Aeusserungsweise 3 verschiedene Arten von Freiheit: 1. die F. der Bethätigung (libertas exercitii), wobei nur zwischen Handeln oder Nichthandeln gewählt wird. 2. die F. der Art der Bethätiguing (l. specificationis), d. i. die Wahl zwischen einer Handlung und einer andern oder auch ibrem Gegenteil; darunter fällt 3. die F. des Gegenteils (l. contrarietatis), d. i. speciell die Wahlfreiheit zwischen dem sittlich Guten und Bösen. (Kgl. Cathrein, a. a. 0. S. 27). Locke beschränkt hier die Freiheit auf den 1. Fall, ohne dies ausreichend zu begründen. 
er nicht vernag; sondern er ist deshalb frei, weil er die Macht hat zu springen oder nicht zu springen" (\$27).

Wollen aber ist ein Akt der Seele, insofern sie ihr Denken auf Hervorbringung einer Handlung richtet und dabei ihre Macht zu deren Hervorbringung ausübt (wobei unter Handlung auch die Unterlassung einer solchen zu verstehen ist) ( $\$ 28)$. Wille ist also die Seelenkraft, die die wirkenden Vermögen zur Bewegung oder Ruhe bestimmt, so weit sie von einer solchen Bestimmung abhängig sind ( $\$, 29)$.

Der obigen Frage nun, ob Freiheit darin bestehe, wie der Willensakt ausfällt, könnte man auch die Fassung geben: was bestimmt den Willen gerade so $\mathrm{zu}$ wollen, wie er will. Darauf ist die wahre und passende Antwort: die Seele. Denn das, was die allgemeine bestimmende $\mathrm{Kraft}$ (d. $\mathrm{i}$. den Willen) zu dieser oder jener besonderen Bestimmung leitet, ist nur das Wirkende selbst, das seine Kraft in dieser besonderen Richtung ausübt. (For that which determines the general power of directing to this or that particular direction, is nothing but the agent itself exercising the power it has, that particular way.)

6. Locke erkennt aber, dass diese Antwort nicht völlig befriedige, dass man vielmehr weiter fragen könne: was veranlasst die Seele ihre Kraft in dieser oder jener besonderen Richtung auszuüben? - worauf wohl auch eigentlich die Frage ziele: was bestimmt den Willen? (What determines the will? § 29.)

In der That ist Locke jetzt bei dem eigentlichen Problem der Willensfreiheit, bei der Frage nach dem Verhältnis der Motive zu den Willensakten, angelangt. Es ist charakteristisch für seine Behandlung dieses Problems, dass er hier über die Ansicht des Indeterminismus zunächst stillschweigend hinweggeht (erst in anderem Zusammenhange ( $\$ 48$ ff.) setzt er sich - mehr gelegentlich - damit auseinander), dass ihm vielmehr die durchgängige Determination des Willens von vornherein feststeht.

Die Antwort nun auf die obige Frage, was den Willen bestimme, lautet: der Beweggrund zum Verharren in einem Zustand oder einer Handlung ist Befriedigung, der Beweggrund zu einer Aenderung ist Unbehagen (The motive for continuing in the same 
state or action, is only the present satisfaction in it; the motive to change, is always some uneasiness. $\S 29$ ).

Locke fasst im folgenden zunächst lediglich die Veränderung, die Hervorbringung eines neuen Handelns ins Auge, und so kann er auch kurzerhand erklären: das Unbehagen ist das, was den Willen bestimmt. (Uneasiness determines the will. $\$ 31$ ).

Mit dem Unbehagen ist nämlich immer ein Begehren (desire) gegeben $(\$ 31)$ - deshalb ist in $\S 30$ will und desire nochmals von einander geschieden - und zwar sind beide untrennbar, so dass 7. B. ein abwesendes Gut so lange nicht unser Begehren erregt, als nicht sein Mangel als Unbehagen empfunden. wird. (Vgl. II, 20, \$6).

Umgekehrt ist auch mit jedem Begehren ein Unbehagen gegeben, und dies ist gerade das Wirksame in dem Begehren, was den Willen bestimmt ( $(32)$. Dieser Satz wird nun aus der Erfahrung ( $\$ 34$ u. 35) und aus der Natur der Sache ( $\$ 36-38)$ dargethan.

Die Erfahrung zeigt, dass, wenn jemand von allem Unbehagen frei d. h. ganz mit seinem Zustand zufrieden ist, sein ganzes Wollen und Handeln darauf gerichtet ist, in diesem Zustand zu verharren, es kommt also nicht zu einer Aenderung, zu einem neuen Wollen und Handeln - was Locke hier allein - (ausser in $\S 39$ i. f.) in Betracht zieht. Eben deshalb hat z. B. Gott das Unbehagen des Hungers und Durstes und anderer natürlicher Begierden in uns gelegt, weil er erkannte, dass der Mensch nur dadurch zum thätigen Wesen werde und sich und die Gattung erhalte $\left.^{52}\right)(\S 34)$.

Ferner beweist die Erfahrung, dass die allgemeine Ansicht (der

52) Er fügt bei: „Ich möchte glauben, dass, wenn die blosse Betrachtung dieser guten $Z$ wecke, zu denen diese mancherlei Unbehaglichkeiten treiben, genügt hätte, um den Willen zu bestimmen und uns zum Handeln zu Veranlassen, wir keine dieser natürlichen Schmerzen und vielleicht in dieser Welt nur wenig oder gar keine Schmerzen haben würden." Wie bezeichnend für seinen gläubigen Optimismus!

Die Bedeutung der von Locke so nachdrücklich verfochtenen Ansiģht, dass lediglich das Unbehagen den Willen bestimme, und die Art seiner Beweisführung soll unten gewürdigt werden. 
sich Locke selbst in der ersten Auflage angeschlossen hatte), das grösste Gut bestimme den Willen, unhaltbar ist. Denn dieses erregt, trotzdem wir es kennen und als Gut anerkennen, den Willen nicht, solange nicht das ihm entsprechende Begehren ein Unbehagen über dessen Mangel erweckt. An mehreren Beispielen sucht Locke dies zu zeigen, um schliesslich auf den Satz hinzuweisen, in dem diese vielfache Erfahrung sich ausspreche: video meliora probogue, deteriora sequor ( $(35)$.

Eine Erwägung der Natur der Sache führt zu dem gleichen Resultat. Unser Streben geht bei all unserm Thun auf Glück, nun verträgt sich aber Unbehagen und Glück nicht, ein kTeiner Schmerz genügt, alle Freude zu zerstören. So wird also das Unbehagen und damit das Begehren nach Beseitigung desselben den Willen bestimmen, solange noch ein solches vorhanden ist - und dies ist nach Lockes Voraussetzung ( $\$ 45)$ fast stets der Fall (\$36).

Ferner ist das Unbehagen allein gegenwärtig, es kann also allein auf den Willen wirken: Abwesendes, wie etwa ein entferntes Gut, kann da nicht wirken, wo es nicht ist. Wenn man einwende, die Betrachtung mache das abwesende Gut zu einem gegenwärtigen, so sei zu beachten: „Solange die blosse Vorstellung eines Gutes in der Seele ist, bleibt sie wie andere Vorstellungen nur Gegenstand unthätiger Betrachtung, wirkt nicht auf den Willen und treibt nicht zur That" (\$37). Zum Beleg hierfür weist er hin auf die schwache Wirkung, die die Vorstellung der ewigen Seligkeit auf das Handeln der meisten Menschen ausübe, und auf die Erfahrungsthatsache, dass kein erhebliches Unbehagen unbeachtet bleibe, sondern den Willen gewissermassen keinen Augenblick loslasse ( $§ 38$ ).

Zur abschliessenden Begründung seiner These, dass lediglich das Unbehagen den Willen bestimme, hebt Locke noch hervor, dass nicht nur im Begehren (was bisher allein betrachtet wurde), sondern aucb in den anderen Gemütserregungen, die auf den Willen wirkten, wie Zorn, Abscheu u. s. w. ein Unbehagen (und damit allerdings auch ein Begehren) gegeben sei, und dass dieses gerade in ihnen das Wirksame sei. Sein Satz gelte aber auch für das auf Erhaltung eines gegenwärtigen behaglichen Zustands gerichtete 
Wollen und Handeln, denn , selbst in der Lust ist das, was die Thätigkeit aufrecht erhält, von der die Lust bedingt ist, das Begehren (desire) sie zu erhalten und die Furcht sie zu verlieren, “ also ein Unbehagen ( $\$ 39$ ). -

Dic Erwägung, dass stets mancherlei Begehren also auch mancherlei Unbehagen auf den Willen wirke, führt zu der genaueren Formulierung des Satzes: „das drückendste Unbehagen bestimmt natürlich den Willen.“ (§ 40).

7. Es hat sich also als Antwort auf die Frage, was den Willen bestimme, ergeben: das Unbehagen bestimmt ihn und zwar das drückendste Unbehagen. Mit jedem Unbehagen aber ist, wie Locke oben zu zeigen gesucht hatte, ein Begehren (desire) gegeben. Es erhebt sich nun die weitere Frage: was ist der Gegenstand des $\mathrm{Be}$ gehrens? Die Antwort lautet: es ist das Glück (happiness) ( $\$ 41$ ).

Glück ist das äusserste Mass der Lust, dessen der Mensch fähig ist, Elend der äusserste Schmerz $\left.(\$ 42)^{53}\right)$.

Alles, was uns Lust gewährt, heisst und ist ein Gut (good), was uns Schmerz bereitet, ein Uebel (evil). Also kann man auch sagen: der Gegenstand des Begehrens ist im allgemeinen ein Gut.

Aber nicht jedes Gut, auch wenn man es als ein solches anerkennt, erregt - wie schon oben gezeigt - unser Begehren, sondern nur das, das als notwendiger Bestandteil unseres Glückes gilt, so dass also sein Mangel mit Unbehagen empfunden wird. Es kommt mithin hierbei auf die individuelle Beschaffenheit und die damit gegebene Empfänglichkeit der Einzelnen an. Findet der eine seinen Genuss lediglich im Studium, der andere lediglich im sinnlichen Vergnügen, so wird keiner das, worin der andere seine Freude findet, als notwendigen Bestandteil sein es Glückes ansehen, wenn er auch anerkennt, dass darin eine gewisse Lust gefunden werden könne. „So kann man trotz des ernsten und fortwähren-

53) Er fährt fort: „Bei dem Schmerz ist man dagegen allemal beteiligt: man fühlt kein Unbehagen, ohne davon bewegt $\mathrm{zu}$ werden". Es sei hier nur darauf hingewiesen, dass dabei nicht „Gut" und „Uebel“, sondern „Gut" und „Schmerz" (pain) entgegengesetzt werden. Die Scheidung des Vorstellungsund des Gefühlsbestandteils innerhalb des Motivs hätte die Erörterung klarer gemacht. (Vgl. Wundt, Grundriss d. Psychologie. (1896). S. 218.) 
den Jagens nach dem Glück ein grosses und anerkanntes Gut deutlich sehen, ohne davon erregt zu werden, sobald man sein Glück auch ohnedies erreichen zu können glaubt" (§ 43).

Deshalb wird auch das grösste Gut (die ewige Seligkeit) nicht immer begehrt. Einen Beleg dafür bietet die Thatsache, dass die Menschen nicht sterben wollen, obwohl ihnen das Leben im besten Falle nur sehr mässige Genüsse, untermischt mit mannichfachem Unbehagen, bietet, und sie anderseits zugestehen, dass im Jenseits ein Zustand dauernder höchster Freude bestehe (\$ 44). Denn die vielfachen irdischen Bedürfnisse, die teils natürlich, teils durch Mode, Beispiel und Erziehung uns eingepflanzt sind, erregen in uns während des grössten Teils des Lebens eine solche Kette von Unbehaglichkeiten, dass wir mit deren Beseitigung zunächst vollauf zu thun haben und selten frei sind zur Betrachtung entfernter Güter ( $\$ 45)$. -

8. Hier ist nun die Untersuchung, die bis dahin im wesentlichen unter dem Einflusse der empiristisch-nominalistischen Richtung in Lockes Denken stand, auf einem Punkte angelangt, wo ihr seine religiösen und ethischen Grundanschauungen den Pfad, den sie nun einschlagen musste, bestimmt vorzeichneten; ob dies freilich geschehen konnte, ohne die ursprüngliche Richtung zu verlassen, soll zunächst dahin gestellt bleiben.

Die Beobachtung hatte bis jetzt ergeben, dass die Vorstellung des grössten Gutes, der ewigen Seligkeit, auf das Wollen und Thun der Menschen nur sehr geringen Einfluss übt, da sie nur selten ihr Begehren erregt. Aber Gott hat doch die Menschen zur Seligkeit bestimmt; sie sollen sie begehren: nur dadurch werden sie veranlasst, das Sittengesetz zu beobachten, worin das unerlässliche Mittel zur Erreichung des ewigen Glückes liegt. Wie kann Gott vollends die Menschen strafen, wenn sie das von ihm gesetzte Ziel verfehlen, da, wie die seitherige Betrachtung gezeigt hat, es geradezu das Natürliche ist, wenn sie über dem fast unaufhörlichen Unbehagen, das das Leben mit sich bringt, nicht zu einem Begehren des höchsten Gutes kommen?

Locke findet jedoch einen Ausweg aus dieser Schwierigkeit. „Man kann durch eine gehörige Betrachtung und Prüfung eines 
vorgestollten Gutes das Begehren darnach [also nuch ein Unbehagen!] in einem, scinem Werte entsprechenden, Grade erwecken, und dadurch kann es seincrseits auf den Willers wirken und erstrebt werden" $\left.(\$ 46)^{\text {st }}\right)$. Was giebt aber die Möglichkeit dieser Prïfung? Eben jone Solbstmacht des Menschen, die Freiheit zu handeln oder nicht zu handeln, die sich also hier darin äussert, dass er es vermag die.Befriedigung eines Begehrens (genauer: den dazu erforderlichen Willensentschluss) aufzuschieben, bis man die gehörige Prüfung vorgenommen hat. . Demnach gilt der oben festgestellte Satz, dass das grösste Unbehagen den Willen bestimme; nur mit der hieraus sich ergebenden Einschränkung. Denn durch die auf der Freiheit beruhende Möglichkeit der Prüfung und Ueberlegung ist auch die Möglichkeit geboten, dass die ursprünglichen Stärkegrade der verschiedenen "Unbehagen " verändert werden, dass ein "Gut" infolge der Betrachtung "Begehren" und damit "Unbehagen" errege und zwar in einer solchen Weise, dass dieses nunmehr als stärkstes den Willen bestimmt. So erweist sich ihm also die Freiheit zu handeln oder nicht zu handeln als Quelle desjenigen, was man unpassender Weise „Willensfreiheit“: genannt habe, die eben darin bestehe, dass man in der Lage sei, nach dem Ergebnis eigner Ueberlegung zu handeln. (\$ 47).

9. Der Erörterung dieser "wahren "Willensfreiheit. sind die $\$ \S 47-53$ gewidmet.

Zunächst wird gezeigt ( $\$ \S 48-50$ ), dass in der Bestimmtheit durch das eigene Urteil keine Beschränkung, sondern die wahre Verbesserung der Freiheit liege. Der Mensch als ein von Natur vernünftiges Wesen muss geradezu bei seinem Wollen durch sein Denken und sein Urteil über das Beste bestimmt werden; denn sonst bestimmte ihn ein anderes als er selbst, was die Verneinung der Freiheit wäre. (And therefore every man is put under a necessity by its constitution, as an intelligent being, to. be determined in willing by his own thought and judgment what is.

54) And thus by a due consideration, and examining any good proposed, it is in our power to raise our desires in a due proportion to the value of that good by where in its turn and place it may come to work upon the will, and be pursued. 
best for him to do: else he would be under the determination of some other than himself which is want of liberty. $\$ 48)$.

Auch die freiesten Wesen würden in dieser Weise bestimmt, denn man dürfe behaupten, dass die Engel noch entschiedener als wir in ihrer Wähl des Guten bestimmt seien. Ja, Gott selbst könne das, was nicht gut ist, nicht wählen, damit sei aber doch seine Freiheit vereinbar ( $\$ 49)$ - ganz im Sinne des scholastischen Realismus, der aber gerade dem Menschen auch die Freiheit das Böse zu wählen, zuschrieb. (Vgl. Anm. 51.)

Die Betrachtung des Gegenteils ergiebt dasselbe. „Wenn die Freiheit, die wahre Freiheit darin besteht, dass man sich der Leitung der Vernunft zu entziehen vermag und des Schutzes entbehrt, den uns die Ueberlegung gewährt, dass wir nicht das Schlechtere wählen, dann sind - die Verrückten und die Thoren allein frei:“

Dass wir das Vermögen haben den verschiedenen Begehren gegenüber unseren Entschluss zu suspendiren, ist ein Stillstehen am Kreuzweg, die Ueberlegung ist ein Befragen des Führers, „der Willensentschluss nach dieser Ueberlegung folgt der Anweisung des Führers, und wer nach solcher Anweisung sein Handeln oder Nicht-Handeln einzurichten vermag, ist ein freies Wesen“ ( $\$ 50)$.

Wir sahen: die dem Menschen innewohnende Freiheit bietet ihm nicht nur die Möglichkeit zu handeln, wie er will, sondern auch zu wollen, wie er will, $d$. $h$. der bessere, der vernünftige Teil in ihm, der in der Ueberlegung sich zur Geltung bringt. Worin liegt aber der Grund, dass diese letztere Möglichkeit zur Wirklichkeit werde? In „der Notwendigkeit, das wahre Glück zu suchen" (\$51). Denn „dieselbe Notwendigkeit, welche zur Verfolgung der wahren Seligkeit nötigt, führt auch mit derselben Gewalt zur Hemmung, Betrachtung und Untersuchung der einzelnen Begehren, damit ihre Befriedigung nicht der wahren Glückseligkeit entgegentrete und uns davon ableite“ (\$52). Hierüber später!

Mit einer solchen gründlichen Ueberlegung hat der Mensch seine Pflicht erfüllt. Ist sie nicht möglich - es giebt ja, wie oben gezeigt, Fälle, in denen innere oder äussere Momente die Selbstmacht über unser Denken aufheben - so wird Gott uns ein gnädiger Richter sein. Jedenfalls erwächst dem Menschen die Pflicht 
einerscits an der Mässigung seiner Leidenschaften za arbeiten, damit der Verstand frei prüfen kann; anderseits bemüht zu sein, die Neigungen der Seele durch gehörige Betrachtung dem wahren innerlichen Guten der Dinge anzupassen. Jass wir imstande sind, dieser Pflicht nachzukommen, lehrt die Erfahrung ( $\$ 53$ ).

10. Auf diese Weise glaubt also Locke das Problem, das sich oben ergeben hatte, wie es nämlich den Menschen bei der Mannigfaltigkeit ihrer „Unbehagen“ und Begehrungen, möglich sei, nach dem höchsten Gute za streben, lösen zu können durch die Erkenntnis der "wahren Willensfreiheit". Diese beruhte aber auf der Freiheit $z u$ handeln und auf der „Notwendigkeit das wahre Glück zu suchen" (the necessity of pursuing true hapiness $\S 5$ ó.1). Nunmehr dürfte es aber umgekehrt schwer einzusehen sein, wie es trotz dieser Bedeutung der "Willensfreiheit" komme, dass das Wollen der Menschen so verschiedene Richtungen einschlage. Die Erörterung dieses Problems füllt die $\$ \S 54-68$.

Hier bietet sich als Erklärungsgrund zunächst dieselbe Thatsache, auf die schon (\$43) die Verschiedenheit menschlichen Begehrens hingewiesen hatte: die verschiedene Organisation und Empfänglichkeit der Menschen. Der Geschmack der Seele ist so verschieden wie der des Gaumens: der eine findet sein Glück im Studium und in der Erkenntnis, der andere beim Fischen und Jagen, der dritte in Luxus und Liederlichkeit, und gäbe es kein jenseitiges Leben, so wäre das Glück, das sie hierin finden auch ihr wahres Glück ( $\$ 54$ u. 55).

Nun giebt es aber nach dem Willen Gottes ein einziges, für alle gleichmässig bestimmtes, wahres Glück, und das, was sich oben als Inhalt und Wert der „Willensfreiheit" herausstellte, scheint das Streben nach diesem Glück allen möglich, ja geradezu notwendig zu machen. Locke vergegenwärtigt sich deshalb hier nochmals in Kürze das über die Willensfreiheit Festgestellte. Ihre Bedeutung liegt darin, dass unser Wollen durch das Ergebnis der Ueberlegung bestimmt wird (worauf denn zugleich auch unsere Verantwortlichkeit beruht), aber diese Ueberllegung kan n nachlässig, kann übereilt sein. Hier ist der gesuchte Punkt: dadurch, dass von der Willensfreiheit nicht der rechte Gebrauch ge- 
macht wird, eröffnet sich die Möglichkeit, dass sich die ursprüngliche Verschiedenheit des Begehrens auch trotz der Willensfreiheit, gewissermassen über ihren Wirkungsbereich hinweg, in dem Wollen der Menschen geltend macht ( $\$ 56)$.

Aber da man, wo es sich um Glück und Elend handelt, in der Regel sorgfältig verfährt, so genügt diese eine Erklärung nicht; man muss ausser der verschiedenen natürlichen Disposition auch die verschiedenen "Unbehagen" beachten, die auf den Menschen wirken. Woher diese? ( $\$ 57-68$.)

A. Sie rühren her von körperlichen Schmerzen. Sie heben - wie Locke schon früher ( $\$ 12)$ betont hatte - die Frei-

- heit an anderes zu denken auf. So erscheine es denn begreiflich, dass niemand es vermöge, unter dem Eindruck derselben durch Betrachtung eines zukünftigen Gutes ein Begehren darnach in sich zu wecken, das stark genug wäre, dem aus der körperlichen Qual entstehenden Unbehagen das Gleichgewicht zu halten. So wirkt denn dies letztere übermächtig auf den Willen und lenkt den Menschen ab „von Tugend, Frömmigkeit und Religion und allem, was vorher als zum Glücke führend ${ }^{55}$ ) erachtet worden ist" ( $\left.\$ 57\right)$. B. Sie entspringen einem durch falsches Urteil ${ }^{56}$ ) irre-

${ }^{55}$ ) Es ist bemerkenswerth, wie Lockes hedonistische Grundanschauung, die sich gerade in den letzten Worten so deutlich ausspricht, sich bier unzureichend erweist, Thatsachen des sittlichen Lebens zu erklären, die doch auch ihm nicht unbekannt sein konnten. Treffend hebt hier Kirchmann hervor (Erl. 195): „Es hat zu allen Zeiten Märtyrer gegeben, die, um der Pflicht treu zu bleiben, die höchsten Qualen und den Tad auf sich genommen haben".

56) In der grossen Bedeutung, die Locke im folgenden dem falschen Urteil einräumt, zeigt sich hier auch bei ihm die der damaligen Philosophie überhaupt eigene Ueberschätzung der intellektuellen Seite. Seine ihn in dieser Erörterung leitende Grundanschauung spricht er $\$ 62$ aus: Since I lay it for a certain ground, that every intelligent being really seeks happiness, which consists in the enjoyment of pleasure, without any considerable mixture of uneasiness; it is impossible any one should willingly put into his own draught any bitter ingredient, or leave out any thing in his power, that would tend to his satisfaction, and the completing of his happiness, but only by wrong judgement. - Locke hebt an derselben Stëlle auch hervor, er spreche nicht von dem falschen Urteil, das auf unüberwindlichem Irrtum beruhe, sondern einem solchen, das eine Schuld des Menschen in sich schliesse. 
geleiteten Verlangen nach cinem abwesenden Gut (\$\$ 5868); was zunächst durch cine allgemeinero Betrachtung begründet wird ( $\$ \$ 58-60)$.

Uober das gegen wärtige Gut ist unser Urteil immer richtig. Jie Dinge sind, während man sie geniesst das, was sie scheinen; denn Schmer\% und Isust sind gerade so gross, als man sie fühlt. Schlösse jede Ilandlung mit sich ab und hätte sie keine Folgen, so wïrde man in der Wahl des Guten nie irren. Aber unsere Handlungen haben Folgen, und diese (also zukünftige Güter und Uebel) können Gegenstand der Ueberlegung und des Urteils werden und dadurch auch Gegenstand des Begehrens, das sich ja hiernach von selbst nicht einstellt ( $\$ 59)$. Hieraus ergiebt sich auch die Notwendigkeit erziehlicher Einwirkung. Man muss dem Menschen zeigen, dass Tugend und Religion zu seinem vollkommenen Glück nötig seien: nichts im Leben kann mit dem Glück oder dem Elend der unsterblichen Seele im Jenseits verglichen werden. Bei dieser Einsicht wird der Mensch seine Handlungen nicht mehr durch die kurzen Freuden und Schmerzen dieser Welt bestimmen lassen $(\$ 60)$.

Es folgt nun ( $\$ \S 61-68)$ eine genauere Erwägung der einzelnen Gründe jenes falschen Urteils.

Ein Gut oder ein Uebel ist eigentlich nur Lust oder Schmerz. Weiterhin gilt aber auch alles, was Lust oder Schmerz zur Folge hat, für ein Gut oder für ein Uebel (\$61). Das falsche Urteil greift nun Platz bei den hier nötig werdenden Vergleichungen (\$ 62).

1) Es kann eintreten bei der Vergleichung von Lust und Schmerz selbst: als gegenwärtige erscheinen sie grösser, denn als

Dass sich also die verschiedenen (und verkehrten) Willensrichtungen der Menschen erklären lassen, entschuldigt diese nicht und hebt auch ibre Verantwortlichkeit nicht auf. -

Von der früheren Erörterung über die Bestimmung des Willens durch das Unbehagen ( $\$ \S 29-40)$ unterscheidet sich die hier ( $\$ \S 58-68)$ folgende dadurch, dass hier von dem auf falschem Urteil beruhenden (Begehren und) Unbehagen die Rede ist; denn Begehren nach einem abwesenden Gut (worum es sich hier handelt) und dadurch entstehendes Unbehagen ist ja nach dem früher ( $\$ 46)$ Gesagten nur durch Vermittlung des, auf der Freiheit beruhenden, Ueberlegens und Urteilens möglich. 
zukünftige. 'Wäre der Katzenjammer gleich beim Ergreifen des Glases in seiner Grösse fühlbar, so würde man ihm gegenüber wohl die Lust des Trịnkens gering anschlagen ( $\$ 63)$. Der Grund hierfür liegt in der Enge unseres Bewusstseins: man kann nicht zweierlei Lust gleichzeitig als gegenwärtig und damit in ihrer wahren Grösse empfinden, noch weniger Lust und Schmerz zugleich. Ausserdem erscheint der gegenwärtige Schmerz so gross, dass man vor allem davon los kommen will, mag daraus entstehen, was wolle. Aber auch die Entfernung von gegenwärtiger Lust ist ein Schmerz, der 'uns mächtig zu dem lustbringenden Gegenstand hintreibt (§ 64). : Anderseits wirkt. ein abwesendes Gut, wie oft erwähnt, nur schwach, besonders wenn man es noch nicht selbst gekostet hat, sondern es nur von andern rühmen hört; denn, was andern gẹällt, ist uns oft gleichgültig, und das, woraus wir selbst schon Genuss geschöpft, mag uns zu einer anderen Zeit gar nicht mehr anmuten. - Von dem jenseitigen Glück gilt dies allerdings nicht: das Manna des Himmels wird jedem Gaumen behagen $\left.{ }^{57}\right)(\S 65)$.

2. Ebenso leicht möglich ist das falsche Urteil bei vergleichender.Betrachtung der Folgen der Handlungen. Hier ist man geneigt anzunehmen: a) dass die Folgen weniger schlimm sein werden, als sie wirklich sind; b) dass sie vielleicht gar nicht eintreten werden oder dass man sie durch mancherlei Mittel werde beseitigen können' (\$66). Als weiter zurückliegende Ursachen derartiger falscher Urteile erscheinen: Unwissenheit, Nachlässigkeit, Einfluss von Leidenschaft, Gewohnheit, Mode.

3. Das falsche Urteil kann endlich darin bestehen, dass man das nicht für nötig zu seinem Glücke hält, was es thatsächlich ist. Dazu trägt das wirkliche oder vermeintliche Unangenehme der zur Erreichung jenes Zieles nötigen Handlungen bei $(\S 68)^{58}$ ).

11. Von hier aus leitet dann die Erörterung wieder hin auf

57) Er fügt bei: „Unless they will say: God cannot make those happy he designs to be so". Kirchmann übersetzt: \#.. sofern man nicht sagt: Gott kann jeden nach seinem Belieben glücklicb machen“.

58) Locke kommt in den Ausführungen dieser Punkte vielfach wieder darauf hinaus, dass das falsche Urteil, auf dem die verkehrte Willensrichtung beruhe, eben ein übereiltes sei, was sich mit dem Inhalt des $\$ 56$ deckt. 
eine schon früher ( $\$ 47$ ) berührte Frage: wie nämlich die Versittlichung des Menschen möglich sei ( $\$ 69$ und 70).

Man kann das Angenehme und das Unangenehme an den Dingen verïndern und man soll es. Man kann und soll seinen Geschmack verbessern. Ueberlegung, Uebung, Fleiss, Gewohnheit vermögen es. Die Vernunft rät zum ersten Versuch, die Ausführung versöhnt oft mit dem, was aus der Ferne widerwärtig erschien, und die Gewohnheit verbindet Lust und Behagen so fest mit dem gewohnten Handeln, dass. man es nicht mehr unterlassen kann. Das gilt auch für die Tugend.

Freilich Mode und öffentliche Meinung haben vielfach falsche Ansichten, Erziehung und Lebensweise haben falsche Gewohnheiten verfestigt. Der Geschmack an den Dingen ist dadurch verfälscht. Aber der Einzelne kann dagegen auf dem bezeichneten Wege angehen ( $\$ 69)$.

Zweifellos sollte eine auf ihre wahre Grundlage gebaute Moral Ueberlegung und Wahl bei jedem Verständigen bestimmen. Diese Grundlage aber besteht darin, dass Gott endloses Glück mit einem tugendhaften Leben, endloses Elend mit dem entgegengesetzten verknüpft hat. Wenn dieses ewige Leben auch nur als möglich gilt (und dies kann niemand bezweifeln), so genügt dies, um bei ernster Ueberlegung ein ausreichend starkes Motiv gegen „jede Lust und jeden Schmerz des irdischen Lebens zu bilden". Dazu kommt, dass, alles recht betrachtet, böse Menschen auch hier sich schon in der schlechtesten Lage befinden ${ }^{59}$ ) - ganz abgesehen von der Ewigkeit. Hat aber mit seiner Unsterblichkeitshoffnung der Gute recht, so ist er ewig glücklich; hat er unrecht, so ist er nicht elend: er fühlt nichts. Hat dagegen der Böse recht, so ist er doch nicht glücklich, und hat er geirrt, so ist er unendlich elend (\$ 70). -

12. Es folgt noch in $\S 71$ eine kurze Zusammenfassung. Neu ist hier die (von uns schon früher berührte) Darlegung, in wiefern man die Freiheit auch als eine Unentschiedenheit (indifferency) bezeichnen könne. Er betont, man dürfe diese Indifferenz nicht verlegen zwischen das Urteil (d. i. das Ergebnis der Ueberlegung)

${ }^{59}$ ) Man beachte, wie hier auch die natürliche Verbindung zwischen dem sittlichen Verbalten und Glück oder Unglück anerkannt wird. 
und den Willensentschluss (determination of the will), da dieser jenem unmittelbar folge; aber auch nicht vor die Ueberlegung: „man versetzt damit die Freiheit in einen Zustand der Dunkelheit, in der man nichts von ihr sehen und sagen kann; wenigstens wird sie dann einem Wesen beigelegt, dem die Fähigkeit für sie abgeht, da kein. Wesen der Freiheit fähig ist, wenn man ihm das Denken und Urteilen nimmt.". Auch sei sie nicht sowohl eine Unentschiedenheit des Menschen als vielmehr der wirkenden Vermögen und deren Indifferenz verharre (in dem früher dargelegten Sinne) auch $\dot{n} \dot{a} c h$ dem Willensentschluss.

13. Der Gedankenfortschritt der ganzen Erörterung lässt sich etwa in folgender Weise übersichtlich darstellen:

A Die Vorstellungen "Freiheit" und "Notwendigkeit". $\S 7-13$.

I. Freiheit. $\S 8-12$.

1. Definition der Freiheit als Kraft zu handeln oder nicht zu handeln. $§ 8$.

2. Sie setzt Verstand und Willen und die Abwesenheit innerer und äusserer Hindernisse voraus. § 9-12. (Excurs über die Terminologie. $\S 10$ und 11.)

II. Notwendigkeit. $\S 13$.

B Freiheit kommt in eigentlichem Sinne nicht dem Willen, sondern dem Menschen zu. $\$ 14-21$.

I. Dem Willen kommt die Freiheit nicht zu. $\$ 14$.

II. Nochmalige Erklärung der Begriffe „Wille“ und „Freiheit". $§ 15$. Sie sind Kräfte, die nicht einander, sondern dem Wesen zukommen. § 16.

III. Der Begriff „Vermögen“ (faculty), dessen Gebrauch irreführt, wird erklärt. $§ 17-20$.

IV. Die Freiheit kommt dem Menschen zu. $\$ 21$.

C In welchem Sinne kann etwa Freiheit dem Willen zugeschrieben werden. $\$ 22-70$.

I. Die Freiheit $\mathrm{zu}$ wollen oder nicht zu wollen (meist nicht vorhanden). $§ 22-24$.

II. Die Freiheit $\mathrm{zu}$ wollen, was man will. (Ihre Annahme beruht auf unrichtiger Fragestellung.) $\$ 25-29$, 
III. Die Freiheit des Willens. (Frage nach dem Motiv.) $\S 30-71$.

1. Das Unbohagen bestimmt den Willen. $\$ 30-40$.

a) Mit dem Begehren (das vom Willen zu unterscheiden ist) ist immer ein Unbehagen gegeben, das den Willen bestimmt. $\S 30-32$.

b) Beweis dafür aus der Erfahrung und der Natur der Sache. $\$ 33-38$.

c) Auch mit den anderen Affekten ist ein Unbehagen verbunden. $\$ 39$.

d) Das drückendste Unbehagen bestï̈mt den Willen. $\S 40$.

2) Gegenstand des mit dem Unbehagen verknüpften Begehrens ist Glück (bezw. ein Gut). $\$ 41-45$.

a) Erklärung der Begriffe "Glück“, „Gut" ünd ihres Gegenteils. $\quad \S 41,42$.

b) Welche Güter begehrt werden: $\S 43-45$.

3) Die Disciplinierung des Begehrens.. $\$ 46-70$.

a) Weg zur „wahren Willensfreiheit". Bedeutung derselben. $\$ 47-53$.

b) Erklärung der verschiedenen Willensrichtungen. $\S 54-68$.

a. Die Verschiedenheit der natürlichen Disposition und übereilte Entschliessung. $§ 54-56$.

$\beta$. Die verschiedenen auf den Menschen wirkenden Unbehagen. $\$ 57-68$.

$\alpha^{\prime}$. Körperliche Schmerzen. $\$ 57$.

$\beta^{\prime}$. Durch falsches Urteil irregeleitetes Begehren. $\S 58-68$.

c) Möglichkeit und Pflicht zur Regelung des Begehrens zu gelangen. $\$ 69,70$.

D Zusammenfassung. (Inwiefern kann die Freiheit als Indifferenz bezeichnet werden?) $\$ 71$.

(Schluss folgt.) 